ARTICLE

Received 12 Nov 2015 | Accepted 27 Apr 2016 | Published 17 Jun $2016 \quad$ DOl: 10.1038/ncomms11744

\title{
Floquet topological insulators for sound
}

\author{
Romain Fleury ${ }^{1,2,3, \star}$, Alexander B. Khanikaev ${ }^{4,5, \star} \&$ Andrea Alù ${ }^{1}$
}

The unique conduction properties of condensed matter systems with topological order have recently inspired a quest for the similar effects in classical wave phenomena. Acoustic topological insulators, in particular, hold the promise to revolutionize our ability to control sound, allowing for large isolation in the bulk and broadband one-way transport along their edges, with topological immunity against structural defects and disorder. So far, these fascinating properties have been obtained relying on moving media, which may introduce noise and absorption losses, hindering the practical potential of topological acoustics. Here we overcome these limitations by modulating in time the acoustic properties of a lattice of resonators, introducing the concept of acoustic Floquet topological insulators. We show that acoustic waves provide a fertile ground to apply the anomalous physics of Floquet topological insulators, and demonstrate their relevance for a wide range of acoustic applications, including broadband acoustic isolation and topologically protected, nonreciprocal acoustic emitters.

\footnotetext{
${ }^{1}$ Department of Electrical and Computer Engineering, The University of Texas at Austin, 1616 Guadalupe Street UTA 7.215, Austin, Texas 78701, USA. ${ }^{2}$ ESPCI Paris Tech and Langevin Institute, 1 rue Jussieu, 75005 Paris, France. ${ }^{3}$ Institute of Electrical Engineering, EPFL, Route Cantonale, 1015 Lausanne, Switzerland. ${ }^{4}$ Department of Physics, Queens College of The City University of New York, Queens, New York 11367, USA. ${ }^{5}$ Department of Physics, The Graduate Center of The City University of New York, New York, New York 10016, USA. ${ }^{\star}$ These authors contributed equally to this work. Correspondence and requests for materials should be addressed to A.A. (email: alu@mail.utexas.edu) or to R.F. (email: romain.fleury@espci.fr.) or to A.B.K. (email: akhanikaev@qc.cuny.edu).
} 
$\mathrm{n}$ most acoustic media, time-reversal symmetry requires that sound travels on two-way channels: if a wave can propagate in a given direction, propagation in the opposite direction is also allowed. Therefore, acoustic modes come in pair, and any forward-propagating state is always associated with a backward state at the same frequency. Defects or interfaces that couple forward and backward waves are thus inherently associated with reflections, leading to the central issue of impedance matching, a pivotal problem in the engineering and design of acoustical systems.

The seemingly unrelated field of condensed matter physics offers new solutions to tackle the challenges associated with acoustic impedance matching. Recent years have witnessed the discovery of fermionic condensed matter systems characterized by a unique type of order, topological in nature ${ }^{1-10}$. This discovery did not only expand the existing classification of solid-state systems but also significantly enriched our understanding of quantum and classical phenomena in different branches of physics ${ }^{11-17}$. Topological condensed matter systems feature robust unidirectional bandgap-crossing edge states, offering unusual conduction properties. These edge states exhibit immunity to a broad range of structural imperfections, inherently avoiding backscattering over broad energy ranges and circumventing localization in the presence of disorder ${ }^{18}$. Interestingly, while originally proposed in the context of quantum fermionic condensed matter systems, the concept of topological order also opens a wealth of new possibilities when extended to classical waves ${ }^{14,15,19-24}$ and bosons ${ }^{13,25-29}$. In these systems, which lack the protection available for fermions, topological order emerges from the removal of either time-reversal symmetry ${ }^{13,19,20,22,30-33}$, emulating the Quantum Hall effect, or of certain forms of internal or lattice symmetries $21,23,34-39$, in analogy with topological crystalline insulators ${ }^{40}$. However, only the first approach, which breaks time-reversal symmetry, can guarantee the absence of reflected modes regardless of the nature of the defect. In mechanical systems, topological insulators with strong topological proportion against defects and disorder have been indeed obtained in time-asymmetric gyroscopic systems ${ }^{16,17}$. In fluid acoustics, similar properties can be obtained in networks of acoustic cavities filled with a fluid in motion ${ }^{14,15}$. However, implementing uniform motion in a lattice is challenging, and the inherent losses and noise that intrinsically accompany acoustic propagation in moving media may become detrimental in most application scenarios.

In this article, we solve these issues and enrich the toolkit of acoustic engineering by introducing a nonreciprocal material platform possessing the unique ability to eliminate reflections and impedance matching challenges for sound. This is obtained in an acoustic lattice whose properties are modulated in space and time in a time-harmonic rotating manner, demonstrating the acoustic analogue of Floquet topological insulators ${ }^{41-47}$. Different from time-modulated lattices proposed in photonics ${ }^{32,33,47}$ and phononics $^{48}$, our proposal is based on a slow, on-site rotating modulation scheme, which remarkably, as we prove in the following, does not require phase uniformity across the lattice. This allows us to take a pivotal step towards practical applications of topological insulators for sound. We exploit the fact that the acoustic properties of materials can be modulated in a strong manner, up to tens of percents, compared with electromagnetic properties, opening the possibility to broadband, topologically protected, one-way acoustic devices, including an ultrabroadband acoustic diode that transmits sound only in one direction, and a topologically protected nonreciprocal acoustic emitter. While the concept of Floquet topological insulators was originally introduced in solids ${ }^{41-46}$, in photonics ${ }^{22,32,33,47}$, and recently generalized to the case of photoelastic systems ${ }^{13}$, the theoretical methods used to demonstrate this class of topological order have so far been limited to abstract tight-binding and other idealized models. Thus, even on the theoretical front, Floquet topological order in classical systems has until now evaded rigorous treatment based on first-principle theory and numerical simulations. Here on the contrary we develop and apply a rigorous full-wave treatment to demonstrate the realistic possibility of acoustic Floquet topological insulators and its application to practical devices and new concepts for sound engineering.

\section{Results}

Description of the Floquet acoustic crystal. The system we propose and investigate in the following is shown in Fig. 1a. Consider an acoustic crystal with hexagonal lattice formed by coupled acoustic trimers, connected together along the hexagonal bonds via small rectangular channels. Each trimer can be viewed as a resonant acoustic metamolecule composed of three acoustic cavities coupled by cylindrical waveguides ${ }^{49}$. The acoustic medium filling the crystal is silicone rubber RTV-602, an ultralow loss material ${ }^{50}$ widely used in ultrasonic systems, with density $\rho_{0}=990 \mathrm{~kg} \mathrm{~m}^{-2}$ and compressibility $\beta_{0}=9.824 \times 10^{-10} \mathrm{~Pa}^{-1}$ (ref. 50). Figure $1 \mathrm{~b}$ shows the unit cell of the crystal. The surrounding medium is air, and the cavity diameter is $1 \mathrm{~cm}$ with a thickness of $3 \mathrm{~mm}$, supporting the first dipolar cavity resonance $\sim 60 \mathrm{kHz}$. In the following, we operate well below this cavity resonance, in a frequency range in which each cavity can be modelled as a lumped element storing a net amount of acoustic pressure, described by its acoustic capacitance $C_{0}=\beta_{0} V_{0}$ (ref. 51). A trimer formed by three cavities is, therefore, equivalent to a $L-C$ resonating loop, supporting a doubly degenerate lumped dipolar resonance at $21.6 \mathrm{kHz}$ associated with the resonant exchange of potential and kinetic energy among the cavities and the internal coupling channels (Fig. 1c). The next resonance, occurring at $56.5 \mathrm{kHz}$, is of monopolar nature (Fig. 1d). The trimers are weakly coupled with each other and used as building blocks of the hexagonal lattice. To break time-reversal symmetry in this resonant acoustic metamaterial and to induce topologically nontrivial properties, we modulate the acoustic capacitance of each cavity by a time-dependent on-site potential $\Delta C_{m}(t)=\delta C$ $\cos \left(\omega_{m} t-\varphi_{m}\right)$ enforcing a time-harmonic modulation with strength $\delta C / C_{0}$ and frequency $f_{m}=\omega_{m} / 2 \pi$. The phase $\varphi_{m}$ depends on the considered sub-cavity $m$, so that the modulation imparts an effective spin onto each trimer, breaking time-reversal symmetry. This modulation protocol is for now assumed to be uniform within the crystal, as summarized in Fig. 1b, which defines the modulation strategy over each unit cell. This may be easily achieved in practice by compressing the volume of each cavity using piezoelectric actuators. Modulation up to several tens of percents is possible using suitable actuating strategies, which may be leveraged to further increase the bandwidth of the topologically nontrivial bandgap discussed in the following.

The effect of a weak spatiotemporal modulation on the bulk band structure of the acoustic crystal is shown in Fig. 1e. In the absence of modulation (blue curves), four propagating bands are found $\sim 22 \mathrm{kHz}$ (corresponding to the lumped dipolar resonance of Fig. 1c), along with two high-frequency bands $\sim 56.5 \mathrm{kHz}$ (corresponding to the monopolar resonance of Fig. 1d, see also Fig. 8a in the Methods section). The number of bands is consistent with the fact that the lattice is formed by two metamolecules per unit cell, each providing three degrees of freedom associated with the lowest-order mode of the isolated cavities. Here we focus on the four lower-frequency bands 
$\mathbf{a}$

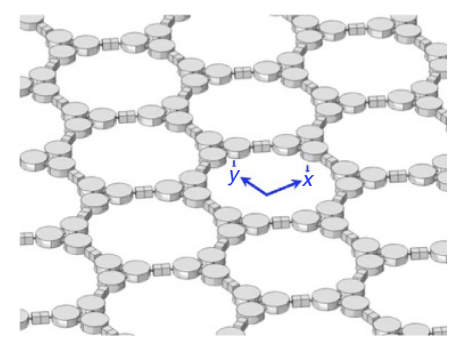

b

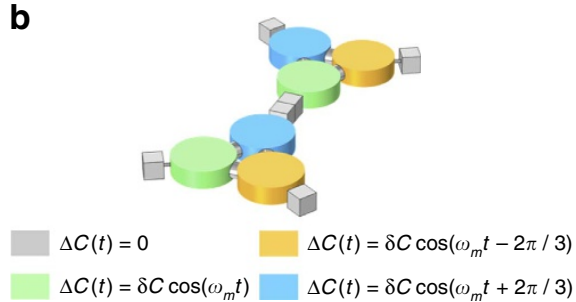

C

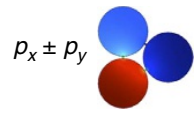

d

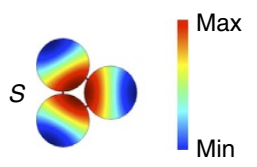

e

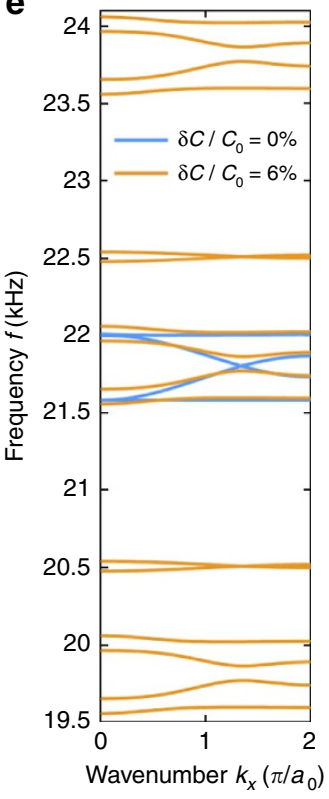

f

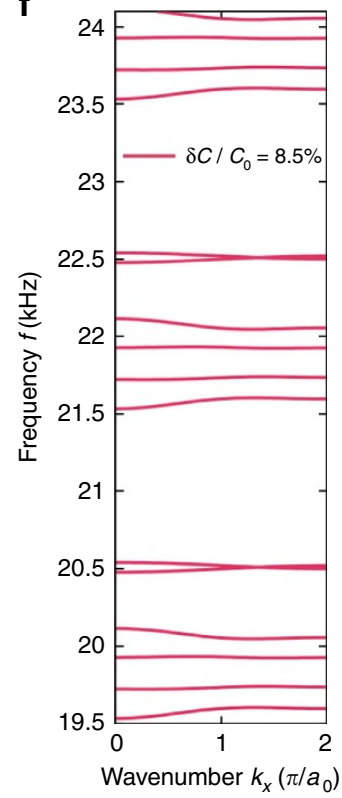

Figure 1 | Hexagonal lattice of modulated acoustic trimers forming an acoustic Floquet topological insulator. (a) We consider a time-dependent phononic crystal formed by a hexagonal lattice of acoustic trimers. The trimers are composed of three interconnected cylindrical cavities (1 cm in diameter), coupled with each other via small rectangular channels. The grey material in the figure is silicone rubber, a water-like ultrasonic material with large-density contrast with surrounding air. In each unit cell (b), the acoustic capacitance $C=C_{0}+\Delta C(t)$ of each cavity is periodically modulated in time at a frequency $\omega_{m}$ and with amplitude $\delta C$ in a rotating fashion, with uniform handedness throughout the lattice. (c,d) First two modes of an acoustic trimer showing the degenerate dipolar lumped resonance $(\mathbf{c})$ at $21.6 \mathrm{kHz}$ and the next dipolar resonance at $60 \mathrm{kHz}$ (d). The colour legend represents the acoustic pressure distribution. (e) Comparison between the bulk band structure in the absence (blue curves) and in the presence (orange curves, $\delta \mathrm{C} / \mathrm{C}_{0}=6 \%, f_{m}=2 \mathrm{kHz}$ ) of modulation. The time modulation has the effect of folding the band structure along the frequency axis and lifting the degeneracy at the $\mathrm{K}$ and $\Gamma$ points, opening a complete bandgap with topological protection. (f) Optimal gap opening occurs at $\delta C / C_{0}=8.5 \%$, for $f_{m}=2 \mathrm{kHz}$.

possessing a dipolar pressure-field profile (corresponding to angular momentum $l=+1,-1)$, which perceive an effective angular-momentum bias $^{52,53}$ provided by the chosen form of spatiotemporal modulation. Two of these bands appear to be slow in nature, corresponding to deaf modes with vanishingly small group velocity, whereas the other two correspond to fast Dirac bands, with degeneracy at both $\Gamma$ and $\mathrm{K}$ points, due to the time-reversal $T$ and combined parity-time-reversal PT symmetry properties of the lattice, respectively. When modulation is applied (orange curves, obtained for $\delta C / C_{0}=6 \%$, $f_{m}=2 \mathrm{kHz}$ ), the band structure folds along the frequency axis, with periodicity equal to $\omega_{m}$, consistent with the Floquet-Bloch theorem in time domain. Given the breaking of temporal symmetry, degeneracy is lifted both at $\Gamma$ and $K$ points by an amount proportional to the modulation depth. Note that when temporal modulation is applied, the high frequency bands around $56.5 \mathrm{kHz}$ are folded and enter the frequency range of interest, but they are intentionally detuned from the dipolar modes in our design.

Notably, this phenomenon happens only for dipolar bands, since dipolar modes can be pictured as a superposition of counter-rotating states $p_{x} \pm i p_{y}$, which overlap well with the rotating modulation scheme. On the contrary, the higherfrequency bands possess double degeneracy at K-point and therefore remain unaffected by the modulation, due to the monopolar $(l=0)$ nature of the mode, orthogonal to the modulation. As it can be seen from equation 5 in Methods, in our system there is no interaction among bands of the same Floquet harmonic order, and therefore no band crossing as understood in its conventional from ${ }^{16,54}$ is present. However, the rotation modulation of the system in time allows interaction of different harmonic orders, which effectively leads to crossing between $p_{x}+i p_{y}$ and $p_{x}-i p_{y}$ bands, and $p_{x}-i p_{y}$ and $p_{x}+i p_{y}$ bands of any two nearest harmonic orders. We emphasize that such interaction is not possible in principle in non-time-Floquet systems, like in previous proposals based on rotating fluids ${ }^{14,15}$, as they are described by time-independent equations due to the stationary character of the fluid flow. This aspect highlights the fundamentally different physics at play here.

When the modulation depth is further increased, with $f_{m}$ unchanged, the bandgap between the two inner bands further opens until the two bands may become flat. This special condition, shown in Fig. If (red curves), occurs for $\delta C / C_{0}=8.5 \%$, and it corresponds to the maximal gap bandwidth. Further increase in modulation depth is detrimental to gap opening, due to pinching of the bands at the $\Gamma$ point. It is important to note that we operate here around the lattice resonance, for which the coupling constant $J$, which measures the strength of off-site coupling between trimers, and whose order of magnitude is given by the spectral span of the unmodulated (blue) band structure (a few hundred $\mathrm{Hz}$ in this example), is smaller than the modulation frequency $f_{m}(2 \mathrm{kHz})$. This ensures that no resonant transitions between different Floquet bands can take place, and bands of different origin and different Floquet orders remain isolated from each other. At the same time, this condition ensures that the effect of on-site modulation is enhanced by the resonant nature of the trimers, enabling strong breaking of time-reversal symmetry with a modulation of a few percents only, at a frequency $(2 \mathrm{kHz})$ one order of magnitude smaller than the acoustic frequency $(20 \mathrm{kHz})$. 
Band topology of the Floquet acoustic crystal. The appearance of a bandgap in the spectrum of the driven system does not guarantee its topological nature. A more detailed analysis of the bands and corresponding eigenmodes is required to determine the class of topological order. In general, temporal modulation of two-dimensional systems can lead to topological order of two distinct classes, both supporting edge states: one characterized by a non-vanishing topological invariant of the first Chern class, known as a Chern insulator, the other one with a vanishing Chern number, or anomalous Floquet insulators, when different Floquet orders interact leading to an exchange of topological charges ${ }^{44,46}$. Since our temporal modulation scheme clearly violates time-reversal symmetry, and since the modulation is sufficiently weak to not resonantly couple different Floquet orders, we expect the opened bandgap to have topological nature of the first Chern class ${ }^{44}$. To fully reveal the topological phase induced by the temporally modulated system of Fig. 1, we developed a semi-analytical approach based on the derivation of the effective Hamiltonian of the time-dependent tight-binding model ${ }^{44}$, which allows a traditional characterization of the system in terms of topological invariants. The geometry under analysis can be mapped onto a tight-binding Hamiltonian associated to a hexagonal lattice with nearest-neighbour hopping, which should be modified to account for the internal structure of the metamolecules

$$
\hat{H}(t)=\sum_{m} \hat{\varepsilon}_{m}(t)|\mathbf{m}\rangle\langle\mathbf{m}|+\sum_{m, n}\left(\hat{J}|\mathbf{n}\rangle\left\langle\mathbf{m}\left|+\hat{J}^{*}\right| \mathbf{m}\right\rangle\langle\mathbf{n}|\right),
$$

where $|\mathbf{m}\rangle=\left|m_{1}, m_{2}, m_{3}\right\rangle$ is a vector whose three components correspond to the three acoustic cavities forming each node of the hexagonal array. The time-independent hopping terms are given by a diagonal $3 \times 3$ matrix $\hat{J}_{m}=J \hat{I}$, while the timedependent on-site term assumes the form

$$
\hat{\epsilon}_{m}=\left(\begin{array}{ccc}
\omega_{0}+\delta \omega_{1}(t) & \kappa & \kappa^{*} \\
\kappa^{*} & \omega_{0}+\delta \omega_{2}(t) & \kappa \\
\kappa & \kappa^{*} & \omega_{0}+\delta \omega_{3}(t)
\end{array}\right),
$$

where $\delta \omega_{l}(t)=\delta \omega \quad \cos \quad\left(\omega_{l} t+\phi_{l}\right)$ and $\phi_{l}=(2 \pi) /(3 l)$. The parameters introduced here directly follow from the form of the Hamiltonian: $\omega_{0}$ is the on-site energy, that is, the lowest resonance frequency of the isolated acoustic cavities, $\delta \omega_{l}(t)$ is its temporal modulation and $\kappa$ is the on-site coupling between cavities of the same metamolecule. We discuss this model in more details in the Methods section.

The Hamiltonian (1) defines the evolution operator $\hat{U}(t)=\mathcal{T} \exp \left[-i \int_{0}^{t} \hat{\mathcal{H}}(t) \mathrm{d} t\right]$, where $\mathcal{T}$ is the time-ordering operator. After writing the Hamiltonian (1) in reciprocal space, we can introduce a stroboscopic evolution operator $U(T)$ for every period of the system $t=n T$, and determine the topological class of the system from its time-independent effective Hamiltonian $^{44} \hat{\mathcal{H}}_{\text {eff }}=\frac{i}{T} \log \hat{U}(T)$. We extracted the effective parameters in equations ( 1 and 2 ) directly from full-wave finite-element method simulations to calculate the Chern numbers $C_{n}=\frac{1}{2 \pi} \int_{B Z}\left(\partial_{k_{x}} A_{y}-\partial_{k_{y}} A_{x}\right) \mathrm{d}^{2} \mathbf{k}$ for every band $n$, using the numerically calculated eigenstates $\left|\mathbf{p}_{n}\right\rangle$ of the effective Hamiltonian $\hat{\mathcal{H}}_{\text {eff }}$ and the Berry connection $\mathbf{A}=-i\left\langle\mathbf{p}_{n}\left|\partial_{\mathrm{k}}\right| \mathbf{p}_{n}\right\rangle$. After integration over the entire Brillouin zone, we found that the four bands of interest possess topological indices $C_{n}=\{1,0,0,-1\}$ and, as expected, reversal of the modulation scheme from clockwise to anticlockwise, equivalent to time reversal, causes the reversal of the Chern numbers $C_{n}=\{-1,0,0,1\}$ (see Methods). The system switches from trivial to nontrivial topology as we turn on the modulation, and the modulated system does not exhibit a topological phase transition as in other works, for example, in ref. 16.
Unidirectional edge states. One of the most appealing features of topological Chern insulators, including the one proposed here, is the existence of one-way edge modes at the boundaries between domains of different topology. The number of topological edge states supported by a given interface is dictated, according to the bulk-boundary correspondence principle ${ }^{55}$, by the change of sum of Chern numbers of all the bulk bands of lower frequency across the interface ${ }^{56}$. In Fig. 2, we consider edge modes propagating on the external edges of a crystal with uniform modulation handedness, as well as edge modes that propagate along the boundary between two crystal domains with opposite modulation handedness. In the first case, the considered interface involves a topologically trivial half space, and therefore we expect only one edge mode, regardless of the crystal termination. The domain wall, for which the difference in the sum of Chern numbers equals two, is expected to support two topologically nontrivial acoustic edge modes (Methods).

Figure 2a considers the case of a crystal terminated by a hard-wall boundary condition, which naturally occurs if the crystal is surrounded by an acoustic medium with very different density, such as air. The figure represents the band structure obtained for a $1 \times 6$ supercell, modulated with $\delta C / C_{0}=8.5 \%$ and $f_{m}=2 \mathrm{kHz}$, and terminated at its top and bottom by hard-wall boundary conditions, with periodic boundary conditions along $x$. Figure $2 \mathrm{a}$ focuses on the frequency region of the four bulk bands of interest: the blue dots correspond to bulk modes, which form four bands separated by a gap, the two in the middle being flat, consistent with Fig. 1f. Differently from Fig. 1, however, the truncated structure now supports two distinct modes within each bandgap. Inspection of the mode profile shows that these modes, with positive (green dots) and negative (red dots) group velocity, are localized at the top and bottom boundaries of the supercell, respectively; thus confirming the one-way response and the chiral character of the modes, which is dictated by the modulation handedness. The edge modes here are composed of different Floquet harmonics, due to the time-dependence, which are all confined to the edge of the lattice. Figure $2 b-d$ shows the acoustic pressure distribution of the three dominant frequency harmonics $\left(\omega-\omega_{m}\right.$ for Fig. 2b, $\omega$ for Fig. $2 c$ and $\omega+\omega_{m}$ for Fig. $\left.2 d\right)$ for one edge mode localized at the top boundary.

Figure $2 \mathrm{e}$ considers the case of a domain wall-the interface between two crystal domains with opposite modulation spin. The plot represents the band structure of a $1 \times 6$ supercell with the bottom three cells and top three cells having opposite modulation handedness. The supercell is surrounded by periodic boundary conditions in all directions. According to the bulk-boundary correspondence principle ${ }^{55}$, this sudden change of modulation spin should be associated with a pair of acoustic edge modes in each bandgap region. In addition, the time-reversed boundary, effectively emerging because of the periodic boundary condition at the top and bottom of the supercell contributes to another pair of acoustic edge modes. A total of four acoustic edge modes are therefore expected in each bandgap region for this case, as confirmed by the band structure in Fig. 2e. The four chiral edges modes that appear within each topological bandgaps can be classified according to the sign of their group velocity: the green dots correspond to positive group velocities, while the red dots correspond to negative group velocities. Inspection of the modal profiles confirms that the modes with positive (respectively, negative) group velocity along $x$ are associated with a righthanded $(\mathrm{RH})$ to left-handed (LH) modulation flip boundary (respectively, LH to RH flip), as the boundary is crossed in the $y$ direction. This confirms the prediction that a single boundary hosts two-edge states with unidirectional properties. Figure $2 \mathrm{f}-\mathrm{h}$ shows the pressure-field distributions associated with the edge mode localized at the centre boundary for all three dominant harmonics. 

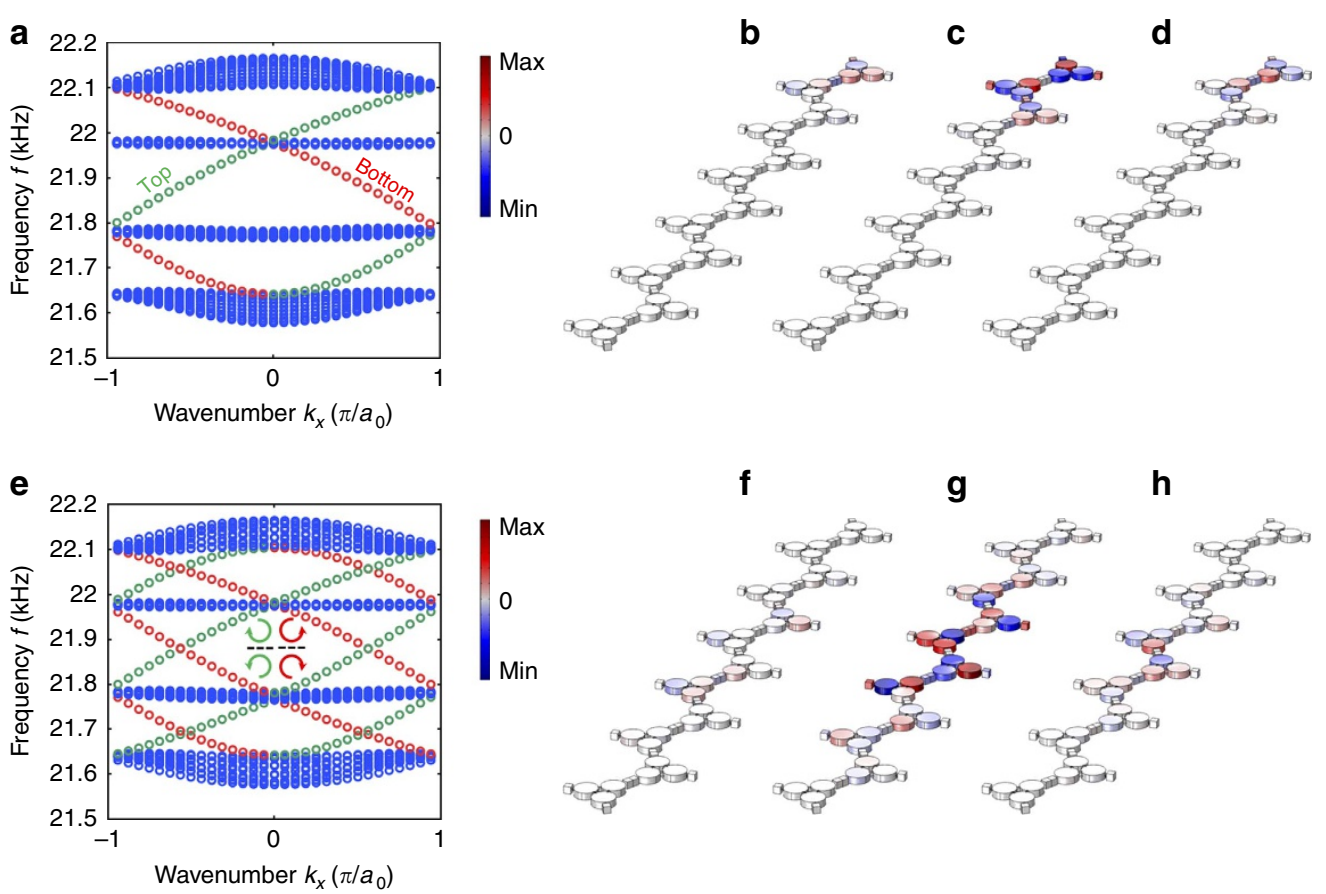

Figure 2 | Topological edge modes of acoustic Floquet topological insulators. (a) Acoustic quasi-band structure for a supercell composed of a 1-by- 6 array of unit cells, terminated by a hard-wall boundary at the top and bottom, and periodic boundary conditions along $x$. Blue dots refer to bulk modes. Green (red) dots refer to edge modes localized at the top (bottom) boundary of the supercell. (b-d) Acoustic pressure-field distribution for the three dominant harmonic components of an edge mode localized at the top boundary: component at $\omega-\omega_{m}$ (b), $\omega$ (c) and $\omega+\omega_{m}$ (d), where $\omega=21.9 \mathrm{kHz}$ is the quasi-frequency. (e) Acoustic band structure for a supercell composed of a 1-by-6 array of unit cells supporting a domain wall at the centre: the modulation of the three unit cells at the bottom is right handed, and at the top it is left handed. The supercell is surrounded by periodic boundary conditions in all directions. Blue dots refer to bulk modes. Red and green dots refer to edge modes localized at boundaries with opposite handedness, as represented in the inset. (f-h) Same as $\mathbf{b}-\mathbf{d}$, but in the case of a domain wall boundary at the supercell centre.

Robustness to structural defects. Breaking time-reversal symmetry in classical and bosonic topological insulators allows for strong topological protection against defects and local disorder ${ }^{20}$. The absence of backward-propagating modes indeed ensures absence of back reflections due to defects and reflections at an arbitrary abruption or discontinuity. To confirm the topological robustness of the Floquet acoustic edge modes, we performed large-scale numerical experiments, deliberately introducing different kinds of lattice defects. Figure 3a shows a drastic example of an acoustic edge state that seamlessly flows along a crystal edge despite the presence of sharp cuts and stringent turns in the hexagonal lattice, and abrupt transitions from zigzag, to armchair, to bearded edge types. Even a severe lattice defect in the form of a missing trimer, placed at the worst location for the edge mode (that is, right on the edge), does not affect one-way propagation. In any natural acoustic material, with topologically trivial properties, the defects would inherently couple forward and backward waves leading to reflections. On the contrary, the chiral acoustic edge states are backscattering immune and exhibit strong protection against any kind of defect, providing robust and reconfigurable propagation over a broad bandwidth. Figure $3 \mathrm{~b}$ shows the case of a chiral mode localized inside a cavity defect, which is dual to that localized at an external edge. Unlike modes localized to a defect in a topologically trivial bandgap material, such a topologically protected spinning mode can only carry positive angular momentum, and its frequency does not depend on the cavity shape, but solely on its perimeter and the topological properties of the surrounding lattice.

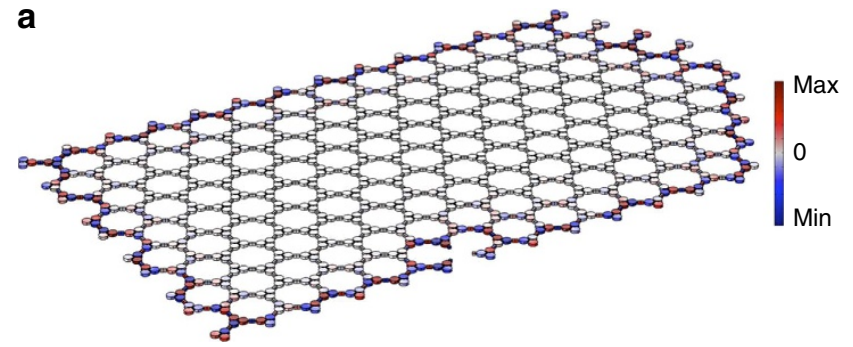

b

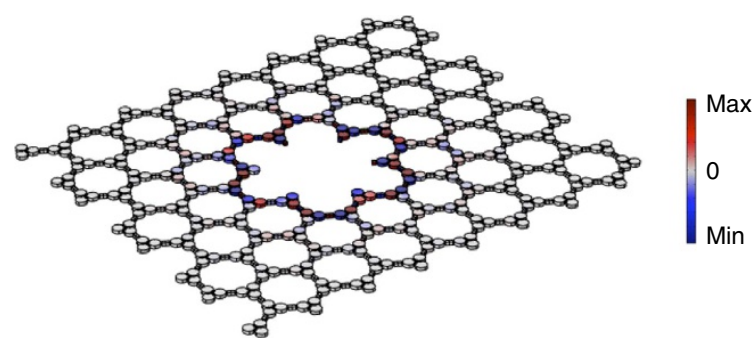

Figure 3 | Strong topological protection of acoustic chiral modes against defects. (a) Immunity of the nonreciprocal edge mode to various defects and turns. The one-way edge states flows seamlessly, regardless of the edge geometry, shape of the turn or the presence of localized defects. (b) Example of uni-rotational acoustic mode localized to a crystal defect in an inner edge. The colour map represents the acoustic pressure. 

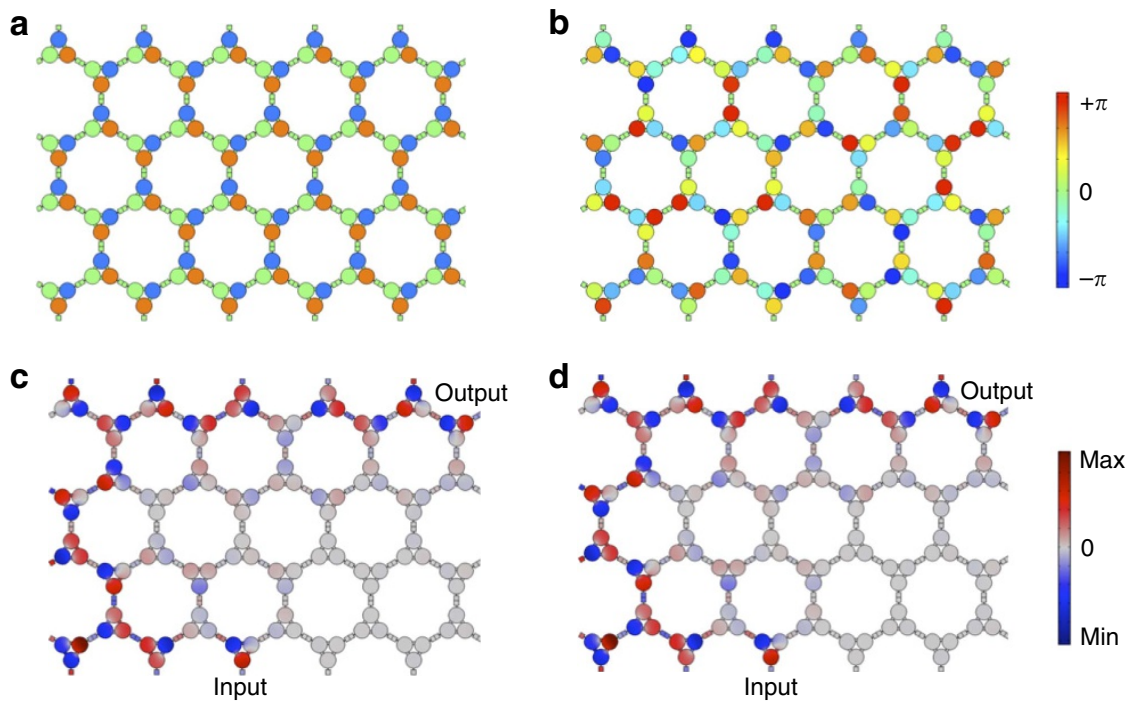

Figure 4 | Robustness to modulation phase disorder. When the modulation is uniform within the crystal (modulation phase shown in a), each trimer is driven with the same phase, and transmission between two points via the edge mode is topologically protected (acoustic pressure, $\mathbf{c}$ ). Remarkably, this behaviour is not affected if each trimer is independently modulated with a random modulation phase (b), and the same acoustic edge mode is excited, both in amplitude and phase (d).

Robustness to modulation disorder. The acoustic edge states supported by acoustic Floquet topological insulators are not only robust against structural defects but also to disorder in the modulation scheme, whose phase can vary arbitrarily from site to site. We demonstrate this extraordinary property using the input-output numerical experiment shown in Fig. 4. First, we consider a perfectly ordered modulation scheme, represented in Fig. 4a. The subcavities composing a given trimer are modulated with $2 \pi / 3$ phase shifts, and the modulation scheme is homogeneous from trimer to trimer. This ideal situation is compared with the largely disordered scenario of Fig. $4 \mathrm{~b}$ in which each trimer is still modulated in a rotating manner, but an arbitrary randomly generated phase is added from trimer to trimer. The plots in Fig. 4c,d show that the acoustic pressure distribution in each case is identical both in magnitude and phase, demonstrating that topological protection is not affected at all by modulation disorder within the lattice. It is only the handedness of the modulation within the trimers that defines the topological properties of the crystal. Thus, our modulation scheme does not have to be uniform throughout the crystal, contrary to previous works on time-dependent photonic lattices ${ }^{32,33,47}$. This major difference and important advantage stems from the fact that these previous proposals have been based on modulating the off-site part of the Hamiltonian, which entangles the phase of the modulation with the Bloch phase, leading to stringent constraints on modulation phase uniformity across large distances. Our proposal is fundamentally different: we modulate the on-site part of the Hamiltonian (the matrix $\hat{\epsilon}$ in (1), which does not depend on the Bloch vector), thus decoupling the effect of the modulation from the phase of the Bloch state. This is allowed by the resonant nature of our trimers that guarantees that the on-site energy is indeed confined within the resonators. With this remarkable property, our proposal constitutes a key step towards the practical implementation of robust, large-scale, Floquet topological insulators, even beyond this acoustic implementation.

A broadband acoustic diode. In addition to robustness against defects and irregularities in geometry or modulation scheme, the proposed acoustic Floquet topological insulators exhibit unique waveguiding properties that enable unprecedented control over wave propagation, leading to a plethora of new potential applications. As seen in Fig. 5a, by tailoring line boundaries of the domain walls within a lattice with opposite modulation spin on each side of the boundary, it is possible to create dynamically reconfigurable, backscattering immune, broadband nonreciprocal waveguides. Here we have imparted a domain wall boundary to control reflectionless routing of an acoustic edge mode between port 1 and port 2 . Inside the crystal, the mode excited at port 1 travels seamlessly to port 2 along the domain wall, regardless of sharp turns and defects along its irregularly shaped path, confirming the possibility to dynamically control and route acoustic signals by controlling the modulation spin, avoiding back reflections. Remarkably, this nonreciprocal reconfigurable waveguide can be used to build a broadband acoustic diode, that is, a device that transmits acoustic waves in the forward direction $1 \rightarrow 2$, but not in the reverse direction $2 \rightarrow 1$, over a continuous, large range of frequencies. Indeed, as seen in Fig. $5 d$, the forward acoustic power transmission coefficient $T_{1 \rightarrow 2}$ (blue solid curve) is unitary over a wide frequency range, only vanishing at a few isolated frequency points corresponding to the bulk crystal bands (blue points in Fig. 2e), whereas transmission in the reverse direction $T_{2 \rightarrow 1}$ (red solid curve) is always negligible. This wideband diode-like behaviour is not a trivial effect that one can attribute to the nonreciprocal response of a single trimer, which would be inherently limited to a single frequency point ${ }^{49,52}$, but rather to a complex lattice effect intimately related to the topologically nontrivial nature of wave propagation. To illustrate this point, we plot the transmission coefficients $T_{1 \rightarrow 2}$ and $T_{2 \rightarrow 1}$ for a single trimer for comparison. It is clear that, even if the single trimer can be used as an acoustic diode (Fig. 5b,c) capable of largely breaking reciprocity, it only allows to do so over a bandwidth of zero measure, that is, at a single frequency, and with $T_{1 \rightarrow 2}<1$. Conversely, when the trimers are assembled in a lattice with a domain wall type of topological boundary, collective effects induce perfect $\left(T_{1 \rightarrow 2}=1, T_{2 \rightarrow 1}=0\right)$ diode-like behaviour over a wide and customizable frequency range (Fig. 5d). Unlike previous proposals on nonreciprocal acoustics ${ }^{57,58}$, the nontrivial topological properties of the lattice induce ideal acoustic diode functionality over a large bandwidth. 


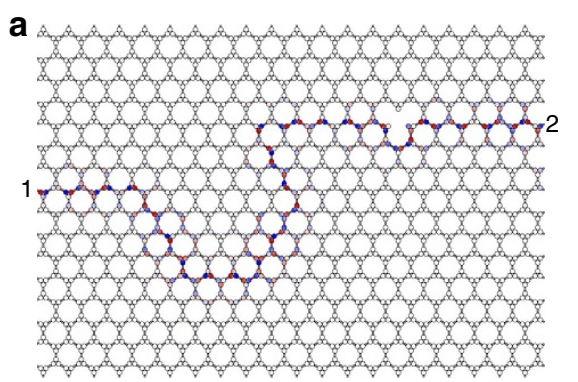

b
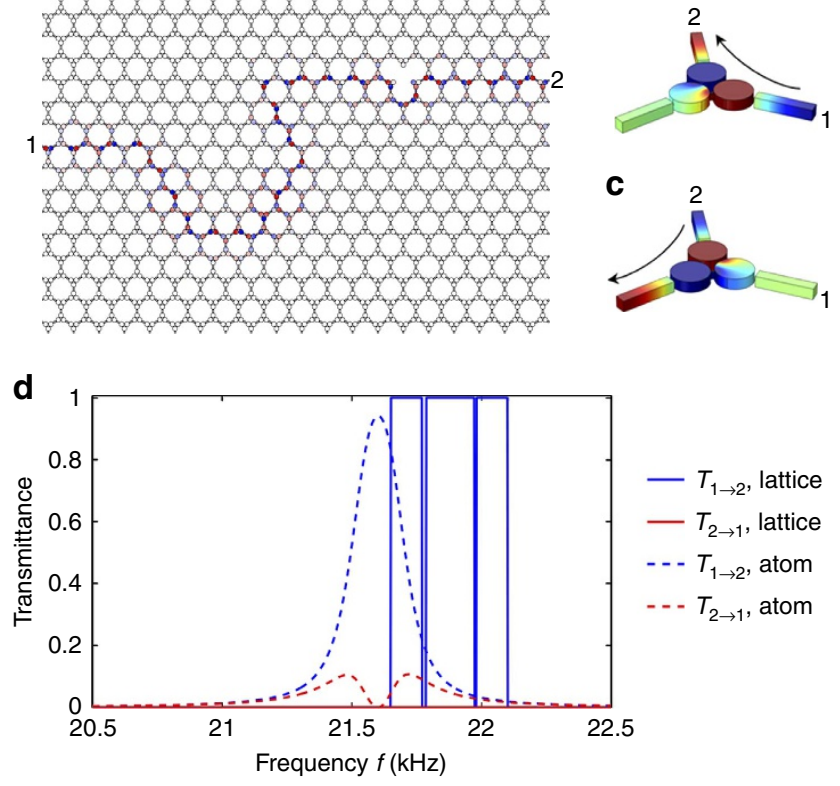

Figure 5 | Broadband topologically protected acoustic diode.

(a) The boundary between two crystal domains with opposite modulation handedness makes a reconfigurable, backscattering immune and broadband nonreciprocal waveguide, which perfectly transmits acoustic waves along the boundary, from the input port 1 to the output port 2 , regardless of turns and defects. This path for the acoustic signal is a reconfigurable one-way channel, and transmission from 2 to 1 is zero, realizing an acoustic diode. A single trimer can also make a diode, transmitting sound from port 1 to 2 (b), but not from 2 to 1 (c).

(d) Frequency dependence of the acoustic power transmission coefficient from 1 to 2 ( $T_{1 \rightarrow 2}$, blue curves) and from 2 to $1\left(T_{2 \rightarrow 1}\right.$, red curves) in the case of the lattice acoustic diode in a (solid lines), demonstrating broadband isolation. For comparison, the single trimer $(\mathbf{b}, \mathbf{c})$ behaves like an imperfect acoustic diode operating only in the vicinity of a single frequency (dashed lines).

Topologically protected acoustic leaky-wave antenna. Topologically protected edge modes may also occur in the presence of radiative losses, when coupling with the radiation continuum takes place at the lattice boundary. This interesting possibility may be exploited in a variety of applications involving acoustic radiation, including underwater acoustic communications systems, acoustic detection and imaging. In Fig. 6, we consider a topologically protected acoustic leaky-wave antenna built by allowing a crystal edge mode to radiate in water, while the bottom and side boundaries are hard-wall boundaries (for example, interfaced with air). The input (feeding) point is located at the bottom edge. One of the practical challenges in conventional leaky-wave antennas is that, if the antenna is truncated before all the energy is radiated out, back reflections cause the appearance of an unwanted beam in the specular direction, impacting directivity and sensitivity to noise. Conversely, as seen in the figure, the proposed topological leaky-wave antenna not only is capable of beam scanning the acoustic radiation both in the forward (Fig. 6a) and backward directions (Fig. 6b) by operating at different frequencies, but the radiation pattern remains highly directional even though the antenna is truncated before all the energy is radiated out, due to the intrinsic absence of a backward mode. Thanks to topological protection, all the power that is not radiated is re-routed at the back of the system and recycled. Different from conventional leaky-wave antennas, which lose
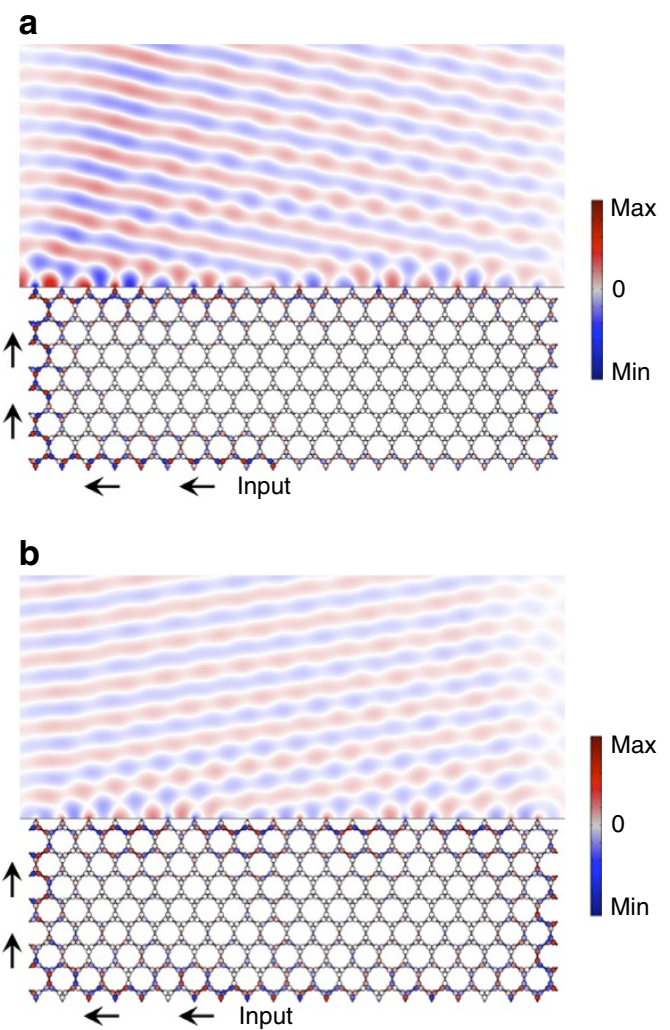

Figure 6 | Topologically protected leaky-wave acoustic antenna.

Topological edge states (acoustic pressure) are robust also in the presence of radiative loss, enabling nonreciprocal leaky-wave radiation from a crystal edge into a surrounding water domain, both in the forward (a) and backward (b) directions, for two different frequencies. Interestingly, such an acoustic leaky-wave antenna may enable 100\% efficiency, regardless of aperture truncation.

their functionality and efficiency on size reduction, radiation efficiency here may become $100 \%$ regardless of truncation, and the antenna does not have impedance matching issues. It is also possible to emit at the opposite angle at the same frequency by simply reversing the lattice handedness (not shown for brevity). As another interesting feature, this acoustic emitter fundamentally breaks the reciprocity constraint, dictated by time-reversal symmetry, based on which a good sound emitter is also a good receiver ${ }^{58}$. Due to the one-way properties of the edge mode responsible for radiation, an acoustic signal impinging on the open edge from the same direction towards which the antenna directively emits would not couple into the edge mode, realizing an acoustic emitter that is not prone to noise and back reflection echoes.

\section{Discussion}

We have proven by first-principle simulations that timemodulated acoustic lattices represent an ideal platform to implement the anomalous physics of Floquet topological insulators, taking a pivotal step towards practical applications of topological acoustics. Using a simple but functional design with readily available acoustic materials, combined with an efficient low-frequency on-site modulation scheme, we have demonstrated that topologically nontrivial properties for sound can be readily obtained in practice without requiring uniform phase of the modulation across the lattice, circumventing a 
practical issue that usually drastically complicates and obscures the applicability of time-modulated crystals. The topological state is only dictated by the local rotational pattern of the modulation phase that is delivered to every resonator, and is totally independent on its phase, which can be completely random from resonator to resonator. Compared with the previous proposals based on time-independent systems with rotating fluids ${ }^{14,15}$, Floquet topological insulators are systems for which the energy/frequency is not conserved, leading to the emergence of side bands. It is exactly the transitions (resonant and non-resonant/virtual) between different side bands that lead to topological order. In fact, the rotational pattern of fluid and the rotational pattern of phase modulation is the only thing this work has in common with refs 14,15 . The rotation of fluid has more similarity with the time-reversal breaking by an external time-independent magnetic field, which also results in cyclotron motion of particles responsible for topological order in condensed matter and photonics systems emulating the quantum Hall effect state. This work, on the contrary, takes the idea of Floquet topological insulators from an abstract solid-state concept to a new practical paradigm in acoustics with large application potential, paving the way to new acoustic systems leveraging

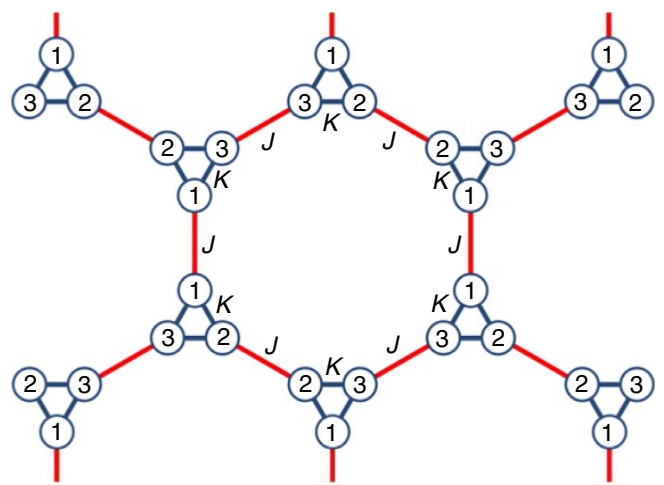

Figure 7 | Hexagonal lattice of the trimer metamolecules. The metamolecules are composed of acoustic cavities indexed as 1, 2 and 3. Blue links show inner connections between the cavities with the strength of coupling (on-site hopping) $\kappa$, and the red lines show the nearest-neighbour (off-site) hopping $J$ between metamolecules. the highly demanded features of topological insulators. The unprecedented broadband nonreciprocal response demonstrated here promises a new class of nonreciprocal elements-acoustic diodes and circulators - which possess unique characteristics of large bandwidth, insensitivity to impedance mismatch and robustness to imperfections in the design implementation. While originally proposed in condensed matter and photonics, Floquet topological insulators may have a surprising twist, finding their first important applications in acoustics.

\section{Methods}

Stroboscopic TB Hamiltonian. As described in the main text, a semi-analytical approach based on the effective Hamiltonian derived with the use of the stroboscopic evolution operator for the time-dependent tight-binding model is used. The Hamiltonian (1) in the main text has a time-modulated on-site part described by the matrix of equation (2), whose diagonal elements are eigenfrequencies of the cavities constituting the metamolecule. These diagonal elements vary with time to form a rotating pattern (that is, $\omega_{l}(t)=\delta \omega \cos \left(\omega_{l} t+\phi_{l}\right)$, $\left.\phi_{l}=(2 \pi) /(3 l), l=1,2,3\right)$, and the off-diagonal components are kept constant. The off-site terms in equation (2) are described with a diagonal matrix, due to the numbering convention shown in Fig. 7.

We show in Fig. 8a the band structure for Hamiltonian (1) without time modulation. The resonant frequency $\omega_{0}$, the coupling parameter $\kappa$ and the hopping parameter $J$ are extracted from full-wave finite-element method numerical simulations performed in COMSOL Multiphysics (acoustic module). As expected, the band structure calculated from the tight-binding Hamiltonian (1) perfectly fits the band structure shown in the main text (Fig. 1e, blue bands), obtained from our numerical experiments.

Next, the time-independent stroboscopic effective Hamiltonian is found as $\hat{\mathcal{H}}_{\text {eff }}=\frac{i}{T} \log \hat{U}(T)$, where $\hat{U}(t)=\mathcal{T} \exp \left[-i \int_{0}^{t} \hat{\mathcal{H}}(t) \mathrm{d} t\right]$ is the evolution operator evaluated over one period. The exponent of the integral in the evolution operator is calculated by numerical integration over one period

$\hat{U}(T)=\mathcal{T} \Pi_{n} \exp \left(-i \Delta t \hat{\mathcal{H}}\left(t_{n}\right)\right)$, where $t_{n}=n \Delta t \in[0, T)$. Here $T$ is the period of modulation, and $\Delta t$ is the time step. Figure $8 \mathrm{~b}$ shows the band structure calculated numerically from the effective Hamiltonian $\hat{\mathcal{H}}_{\text {eff }}$, both without modulation $\delta \omega=0$ (blue bands) and with modulation $\delta \omega \neq 0$ (red bands). One can clearly see the opening of the complete acoustic bandgap predicted by the tightbinding approach, in agreement with the full-wave numerical results shown in the main text (Fig. le,f). Note that by virtue of Floquet theorem, this band structure is periodic along the quasi-frequency axis, and Fig. $8 \mathrm{~b}$ only shows a unit cell of the periodic quasi-band structure, which contains all the information about the system, including about its nontrivial topology.

To confirm the topological nature of the transition from the gapless to the gapped state, we calculated the Berry curvature $\Omega_{n}(k)=\partial_{k_{x}} A_{y}-\partial_{k_{y}} A_{x}$, where $\mathbf{A}=-i\left\langle\mathbf{p}_{n}\left|\partial_{k}\right| \mathbf{p}_{n}\right\rangle$ is the Berry connection and $\left|\mathbf{p}_{n}\right\rangle$ is the eigenstate of the effective Hamiltonian $\mathcal{H}_{\text {eff }}$. The Berry curvature for the four bands of interest calculated at every point in the Brillouin zone is plotted in Fig. 9. The subsequent integration of $\Omega_{n}(k)$ over the Brillion zone yielded the values of the Chern number

$C_{n}=\{ \pm 1,0,0, \mp 1\}$ for the clockwise/anticlockwise modulation of the phase in the trimer metamolecule. Note that the two middle bands (Fig. 9b,c) do not show any
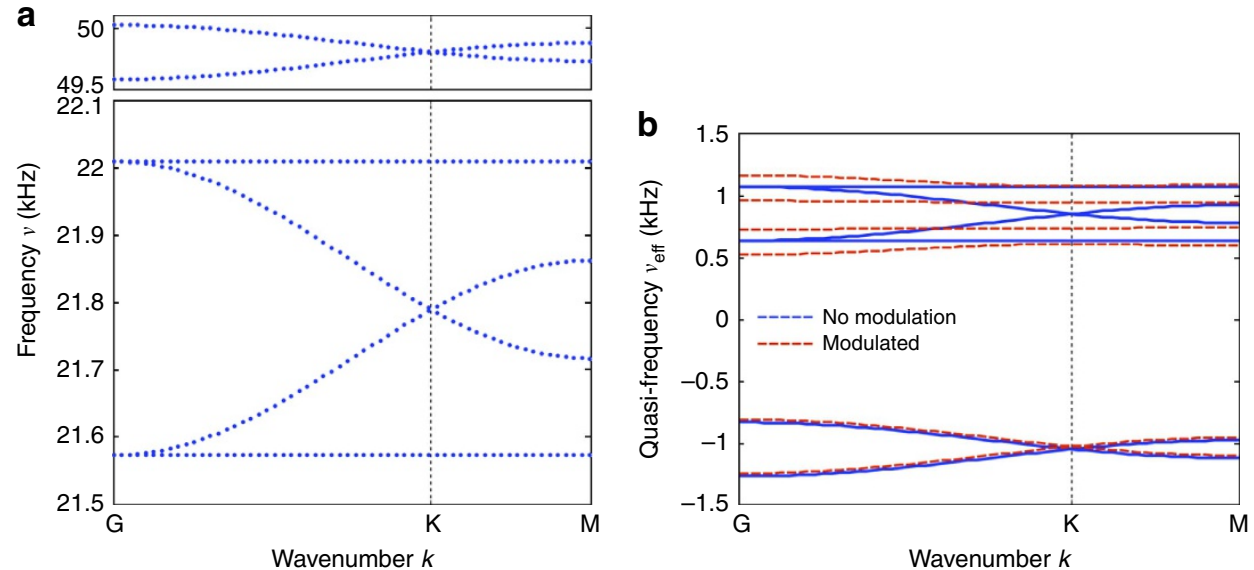

Figure 8 | Analytical acoustic band structure. (a) Acoustic band structure of the tight-binding Hamiltonian equation (1) without temporal modulation $(\delta \omega=0)$. The parameters used are $v_{0}=\omega_{0} / 2 \pi=31.13 \mathrm{kHz}, \kappa=0.3 v_{0}$ and $J=0.7 \times 10^{-2} v_{0}$. (b) Acoustic band structure obtained from the effective Hamiltonian $\hat{\mathcal{H}}_{\text {eff }}$ describing the stroboscopic evolution of the system. Cases without the temporal modulation ( $\delta v=0$, blue solid lines) and with the temporal modulation ( $\delta v=0.036 v_{0}$, red dashed lines) are shown. The parameters used are the same as in $\mathbf{a}$, with the modulation frequency $v_{m}=\omega_{m} / 2 \pi=3 \mathrm{kHz}$. 

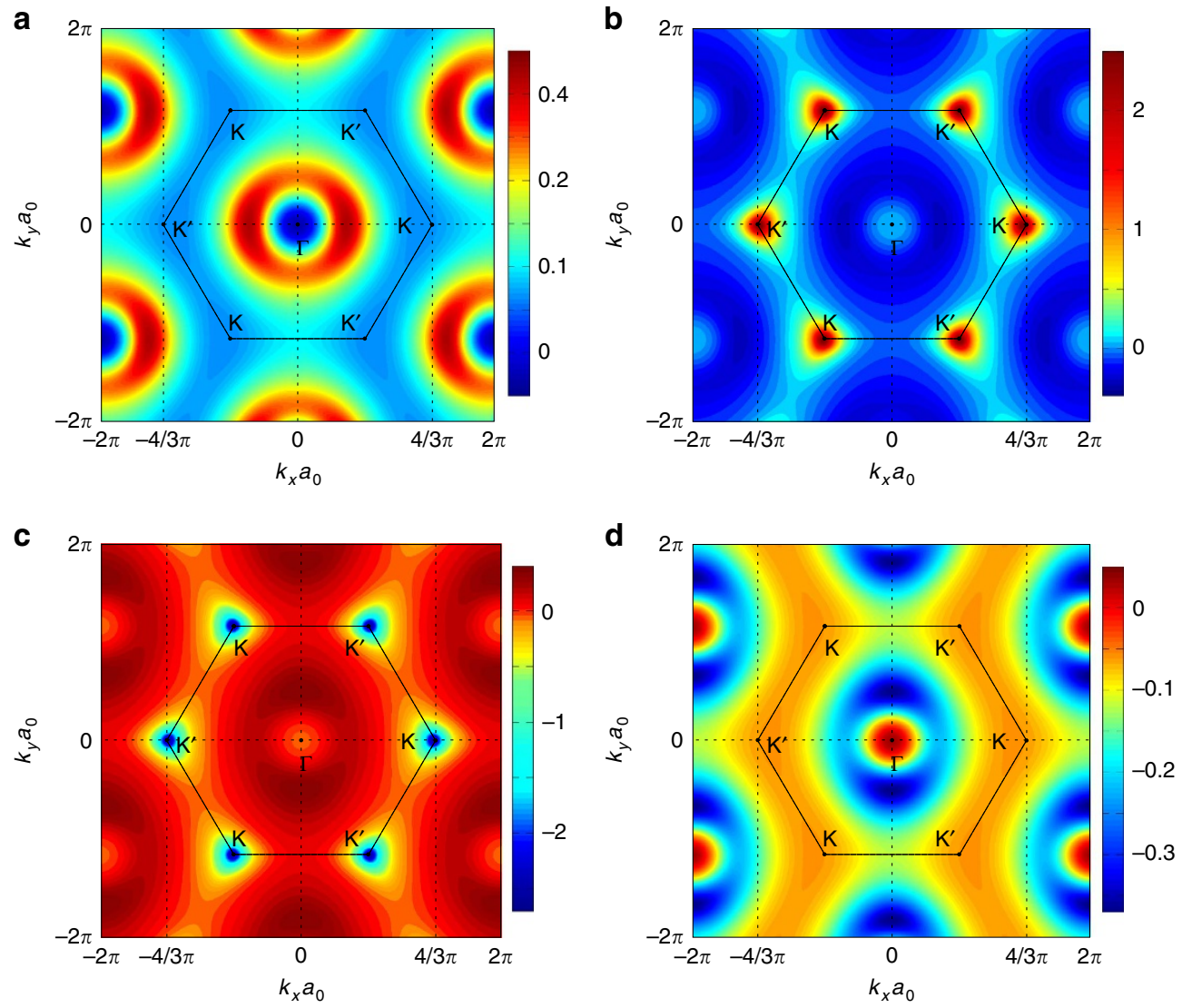

Figure 9 | Berry curvature for the four topological bands of acoustic Floquet topological insulator. The Berry curvature is calculated from the effective Hamiltonian $\hat{\mathcal{H}}_{\text {eff }}$ in the presence of modulation. Subplots (a-d) correspond to the four dipolar bands, from lower to higher frequency, respectively. The parameters used are $v_{0}=\omega_{0} / 2 \pi=31.13 \mathrm{kHz}, \kappa=0.3 v_{0}, J=0.7 \times 10^{-2} v_{0}, \delta v=0.036 v_{0}$ and $v_{m}=\omega_{m} / 2 \pi=3 \mathrm{kHz}$.

topological behaviour, and the Dirac degeneracy at K-point insured by the lattice symmetry persists even in the presence of the modulation. Thus, no topological bandgap is observed for these bands and the corresponding states remain topologically trivial. We can use these values for the Chern numbers of the six bands to predict the number of edge states found at a particular interface. The bulk-edge correspondence principle requires, in principle, to compute the sum of the Chern numbers of all bands below a given gap. In the present case, since the band structure is a periodic repetition of these six bands, which form a 'temporal Brillouin zone', and since the sum of their Chern numbers is always zero, one can always restrict the sum to the six bands belonging to the nearest temporal Brillouin zone ${ }^{46}$. Indeed, the contribution of any full temporal Brillouin zone below the gap to the bulk-edge correspondence principle is zero.

Finally, to demonstrate the presence of an edge state, a lattice supercell was modelled using the effective Hamiltonian obtained from the stroboscopic evolution operator. The supercell consists of 10 unit cells with a domain wall in the centre of the cell, that is, an abrupt flip of the modulation spin. After the time-dependent Hamiltonian for the supercell $\hat{\mathcal{H}}^{\mathrm{SC}}(t)$ was constructed, the procedure was identical to that of the single cell. The eigenmodes of the $\hat{\mathcal{H}}_{\text {eff }}^{\mathrm{SC}}=\frac{i}{T} \log \hat{U}^{\mathrm{SC}}(T)$, with $\hat{U}^{\mathrm{SC}}(t)=$ $\mathcal{T} \exp \left[-i \int_{0}^{t} \hat{\mathcal{H}}^{\mathrm{SC}}(t) \mathrm{d} t\right]$ being the supercell evolution operator, were numerically found. The resultant band structure of the supercell is shown in Fig. 10, and clearly reveals the presence of the four isolated bands (red lines) within the bandgap region induced by the spatiotemporal phase modulation (bulk bands are shown in blue). The inspection of the eigenvectors confirms that these modes are localized to the domain wall, with the edge modes with the positive (negative) group velocity propagating along a $\mathrm{RH} / \mathrm{LH}(\mathrm{LH} / \mathrm{RH})$ boundary, thus confirming their one-way (chiral) character, which is in perfect agreement with the first-principle results presented in the main text.

Finite-element simulations. To perform a full-wave numerical experiment of our time-dependent lattice, we start by noticing that to modulate the cavity capacitance $C_{0}=V_{0} \beta_{0}$ by a relative amount $\delta C / C_{0}$, we can either modulate its volume by an amount $\delta V / V_{0}=\delta C / C_{0}$ or equivalently the compressibility by an amount $\delta \beta / \beta_{0}=\delta C / C_{0}$. In practice, it is naturally easier to modulate directly the volume, by compressing the cavities using piezoelectric actuators. Such a strategy indeed changes the volume without changing the compressibility, as long as linearity holds and Hookes law remains valid. However, for numerical purposes, the capacitance modulation is easier to model via a compressibility modulation, which has the same effect than the volume modulation, but avoids the complexity of a moving mesh and deformed geometry in a numerical code. Therefore, we start from first principles, that is, Euler equation for the motion of a fluid particle and the conservation of mass:

$$
\left\{\begin{array}{c}
\nabla p(\mathbf{r}, t)=-\frac{\mathrm{d}}{\mathrm{d} t}\left(\rho_{0} \mathbf{u}(\mathbf{r}, t)\right) \\
\nabla \cdot \mathbf{u}(\mathbf{r}, t)=-\frac{\mathrm{d}}{\mathrm{d} t}(\beta(\mathbf{r}, t) p(\mathbf{r}, t))
\end{array}\right.
$$

where $p(\mathbf{r}, t)$ is the acoustic pressure, $\mathbf{u}(\mathbf{r}, t)$ is the particle velocity, $\rho_{0}$ is the time-independent density of the medium and

$$
\beta(\mathbf{r}, t)=\beta_{0}+\delta \beta(\mathbf{r}) \cos \left(\omega_{m} t-\varphi(\mathbf{r})\right)
$$

is the dynamically modulated compressibility of the structure. From equation (3), we get the wave equation $\Delta p(\mathbf{r}, t)=\rho_{0} \frac{d^{2}}{d t^{2}}(\beta(\mathbf{r}, t) p(\mathbf{r}, t))$, and use Floquet-Bloch theorem in time domain, writing $p(\mathbf{r}, t)=\sum_{n} f_{n}(\mathbf{r}) e^{i\left(\omega+n \omega_{m}\right) t}$. After a few calculation steps, we obtain the following infinite set of coupled time-independent differential equations for the harmonics $f_{n}(\mathbf{r})$ :

$$
\Delta f_{n}(\mathbf{r})+\rho_{0} \beta(\mathbf{r})\left(\omega+n \omega_{m}\right)^{2} f_{n}(\mathbf{r})=-\frac{1}{2} \rho_{0} \delta \beta(\mathbf{r})\left(\omega+n \omega_{m}\right)^{2}\left\{f_{n-1}(\mathbf{r}) e^{-i \varphi(\mathbf{r})}+f_{n+1}(\mathbf{r}) e^{i \varphi(\mathbf{r})}\right\} .
$$

In our numerical simulations, the relative weakness of the modulation allows solutions to converge quickly, and the system (5) can be truncated to the five harmonics $n=\{-2,-1,0,1,2\}$ that dominate the field in the frequency range of interest. The corresponding equation (5) have been transformed into weak form and directly introduced into the finite-element solver COMSOL multiphysics (acoustic module), where the fully coupled equation system was directly solved in the (quasi-) frequency domain.

Data availability. The data that support the findings of this study are available from the corresponding authors on request. 


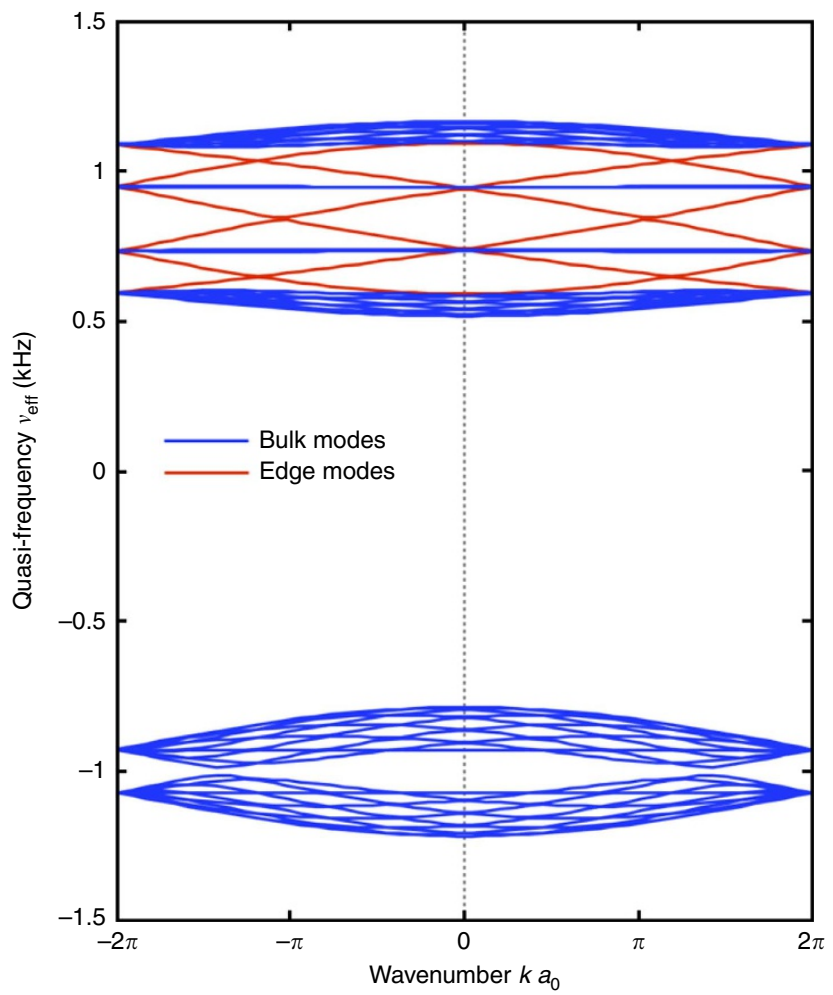

Figure 10 | Acoustic band structure of the $\mathbf{1 0} \times \mathbf{1}$ supercell with domain wall at the centre. The band structure is obtained from the effective Hamiltonian of the stroboscopic evolution. Blue and red lines correspond to the bulk and edge modes, respectively. The parameters used are the same as in Fig. $8 \mathrm{~b}$ in the presence of modulation.

\section{References}

1. Kane, C. L. \& Mele, E. J. $Z_{2}$ topological order and the quantum spin Hall effect. Phys. Rev. Lett. 95, 146802 (2005).

2. Kane, C. L. \& Mele, E. J. Quantum spin Hall effect in graphene. Phys. Rev. Lett. 95, 226801 (2005)

3. Bernevig, B. A., Hughes, T. L. \& Zhang, S.-C. Quantum spin Hall effect and topological phase transition in HgTe quantum wells. Science 314, 1757-1761 (2006).

4. Fu, L., Kane, C. \& Mele, E. Topological insulators in three dimensions. Phys. Rev. Lett. 98, 106803 (2007).

5. Hsieh, D. et al. A topological Dirac insulator in a quantum spin Hall phase. Nature 452, 970-974 (2008).

6. Moore, J. \& Balents, L. Topological invariants of time-reversal-invariant band structures. Phys. Rev. B 75, 121306 (2007).

7. Roy, R. Z2 classification of quantum spin Hall systems: an approach using time-reversal invariance. Phys. Rev. B 79, 195321 (2009).

8. Zhang, H. et al. Topological insulators in Bi2Se3, Bi2Te3 and Sb2Te3 with a single Dirac cone on the surface. Nat. Phys. 5, 438-442 (2009).

9. Hasan, M. Z. \& Kane, C. L. Colloquium: topological insulators. Rev. Mod. Phys. 82, 3045-3067 (2010).

10. Qi, X.-L. \& Zhang, S.-C. Topological insulators and superconductors. Rev. Mod. Phys. 83, 1057-1110 (2011).

11. Lu, L., Joannopoulos, J. D. \& Soljačić, M. Topological photonics. Nat. Photon. 8, 821-829 (2014).

12. Kane, C. L. \& Lubensky, T. C. Topological boundary modes in isostatic lattices. Nat. Phys. 10, 39-45 (2013).

13. Peano, V., Brendel, C., Schmidt, M. \& Marquardt, F. Topological phases of sound and light. Phys. Rev. X 5, 031011 (2015).

14. Yang, Z. et al. Topological acoustics. Phys. Rev. Lett. 114, 114301 (2015).

15. Khanikaev, A. B., Fleury, R., Mousavi, S. H. \& Alù, A. Topologically robust sound propagation in an angular-momentum-biased graphene-like resonator lattice. Nat. Commun. 6, 8260 (2015).

16. Wang, P., Lu, L. \& Bertoldi, K. Topological phononic crystals with one-way elastic edge waves. Phys. Rev. Lett. 115, 104302 (2015).

17. Nash, L. M. et al. Topological mechanics of gyroscopic metamaterials. Proc. Natl. Acad. Sci. USA 112, 14495-14500 (2015).
18. Mittal, S. et al. Topologically robust transport of photons in a synthetic gauge field. Phys. Rev. Lett. 113, 087403 (2014).

19. Haldane, F. D. M. \& Raghu, S. Possible realization of directional optical waveguides in photonic crystals with broken time-reversal symmetry. Phys. Rev. Lett. 100, 013904 (2008).

20. Wang, Z., Chong, Y., Joannopoulos, J. D. \& Soljacić, M. Observation of unidirectional backscattering-immune topological electromagnetic states. Nature 461, 772-775 (2009).

21. Hafezi, M., Mittal, S., Fan, J., Migdall, A. \& Taylor, J. M. Imaging topological edge states in silicon photonics. Nat. Photon. 7, 1001-1005 (2013).

22. Rechtsman, M. C. et al. Photonic Floquet topological insulators. Nature 496, 196-200 (2013).

23. Khanikaev, A. B. et al. Photonic topological insulators. Nat. Mater. 12, 233-239 (2013).

24. Prodan, E. \& Prodan, C. Topological phonon modes and their role in dynamic instability of microtubules. Phys. Rev. Lett. 103, 248101 (2009).

25. Metlitski, M. A., Kane, C. L. \& Fisher, M. P. A. Bosonic topological insulator in three dimensions and the statistical Witten effect. Phys. Rev. B 88, 035131 (2013).

26. Chen, X., Gu, Z.-C., Liu, Z.-X. \& Wen, X.-G. Symmetry protected topological orders and the group cohomology of their symmetry group. Phys. Rev. B 87, 155114 (2013).

27. Vishwanath, A. \& Senthil, T. Physics of three-dimensional bosonic topological insulators: surface-deconfined criticality and quantized magnetoelectric effect. Phys. Rev. X 3, 011016 (2013).

28. Senthil, T. Symmetry-protected topological phases of quantum matter. Annu. Rev. Condens. Matter Phys. 6, 299-324 (2015).

29. Ye, P. \& Gu, Z.-C. Vortex-line condensation in three dimensions: a physical mechanism for bosonic topological insulators. Phys. Rev. X 5, 021029 (2015).

30. Raghu, S. \& Haldane, F. Analogs of quantum-Hall-effect edge states in photonic crystals. Phys. Rev. A 78, 033834 (2008).

31. Wang, Z., Chong, Y. D., Joannopoulos, J. D. \& Soljačić, M. Reflection-free one-way edge modes in a gyromagnetic photonic crystal. Phys. Rev. Lett. 100, 013905 (2008).

32. Fang, K., Yu, Z. \& Fan, S. Realizing effective magnetic field for photons by controlling the phase of dynamic modulation. Nat. Photon. 6, 782-787 (2012).

33. Fang, K. \& Fan, S. Controlling the flow of light using the inhomogeneous effective gauge field that emerges from dynamic modulation. Phys. Rev. Lett. 111, 203901 (2013).

34. Hafezi, M., Demler, E. A., Lukin, M. D. \& Taylor, J. M. Robust optical delay lines with topological protection. Nat. Phys. 7, 907-912 (2011).

35. Ma, T., Khanikaev, A. B., Mousavi, S. H. \& Shvets, G. Guiding electromagnetic waves around sharp corners: topologically protected photonic transport in metawaveguides. Phys. Rev. Lett. 114, 127401 (2015).

36. Chen, W.-J. et al. Experimental realization of photonic topological insulator in a uniaxial metacrystal waveguide. Nat. Commun. 5, 5782 (2014).

37. Chen, W.-J., Zhang, Z.-Q., Dong, J.-W. \& Chan, C. T. Symmetry-protected transport in a pseudospin-polarized waveguide. Nat. Commun. 6, 8183 (2015)

38. Lu, L., Fu, L., Joannopoulos, J. D. \& Soljačić, M. Weyl points and line nodes in gyroid photonic crystals. Nat. Photon. 7, 294-299 (2013).

39. Lu, L. et al. Experimental observation of Weyl points. Science 349, 622-624 (2015).

40. Fu, L. Topological Crystalline Insulators. Phys. Rev. Lett. 106, 106802 (2011).

41. Inoue, J. \& Tanaka, A. Photoinduced transition between conventional and topological insulators in two-dimensional electronic systems. Phys. Rev. Lett. 105, 017401 (2010).

42. Otterbach, J., Ruseckas, J., Unanyan, R. G., Juzeliūnas, G. \& Fleischhauer, M. Effective magnetic fields for stationary light. Phys. Rev. Lett. 104, 033903 (2010).

43. Lindner, N. H., Refael, G. \& Galitski, V. Floquet topological insulator in semiconductor quantum wells. Nat. Phys. 7, 490-495 (2011).

44. Kitagawa, T., Berg, E., Rudner, M. \& Demler, E. Topological characterization of periodically driven quantum systems. Phys. Rev. B 82, 235114 (2010).

45. Dóra, B., Cayssol, J., Simon, F. \& Moessner, R. Optically engineering the topological properties of a spin Hall insulator. Phys. Rev. Lett. 108, 056602 (2012).

46. Pasek, M. \& Chong, Y. D. Network models of photonic Floquet topological insulators. Phys. Rev. B 89, 075113 (2014).

47. Oka, T. \& Aoki, H. Photovoltaic Hall effect in graphene. Phys. Rev. B 79, 081406 (2009).

48. Swinteck, N. et al. Bulk elastic waves with unidirectional backscatteringimmune topological states in a time-dependent superlattice. J. Appl. Phys. 118, 063103 (2015).

49. Estep, N. A., Sounas, D. L., Soric, J. \& Alù, A. Magnetic-free non-reciprocity and isolation based on parametrically modulated coupled-resonator loops. Nat. Phys. 10, 923-927 (2014). 
50. Folds, D. L. Speed of sound and transmission loss in silicone rubbers at ultrasonic frequencies. J. Acoust. Soc. Am. 56, 1295-1296 (1974).

51. Blackstock, D. T. Fundamentals of Physical Acoustics (John Wiley \& Sons, 2000).

52. Fleury, R., Sounas, D. L., Sieck, C. F., Haberman, M. R. \& Alù, A. Sound isolation and giant linear nonreciprocity in a compact acoustic circulator. Science 343, 516-519 (2014).

53. Fleury, R., Sounas, D. L. \& Alù, A. Subwavelength ultrasonic circulator based on spatiotemporal modulation. Phys. Rev. B 91, 174306 (2015).

54. Xiao, M. et al. Geometric phase and band inversion in periodic acoustic systems. Nat. Phys. 11, 240-244 (2015).

55. Hatsugai, Y. Chern number and edge states in the integer quantum Hall effect. Phys. Rev. Lett. 71, 3697-3700 (1993).

56. Skirlo, S. A., Lu, L. \& Soljačić, M. Multimode one-way waveguides of large Chern numbers. Phys. Rev. Lett. 113, 113904 (2014).

57. Maznev, A. A., Every, A. G. \& Wright, O. B. Reciprocity in reflection and transmission: what is a 'phonon diode'? Wave Motion 50, 776-784 (2013).

58. Fleury, R., Sounas, D. L., Haberman, M. R. \& Alù, A. Nonreciprocal acoustics. Acoust. Today 11, 14 (2015).

\section{Acknowledgements}

This work was supported by the National Science Foundation with grant no. CMMI-1537932 (to A.A. and A.K.), the Air Force Office of Scientific Research with grants no. FA9550-14-1-0105 (to A.A.) and FA9550-13-1-0204 (to A.A.), and the
Defense Threat Reduction Agency with grant no. HDTRA1-12-1-0022 (to A.A.). R.F. acknowledges the support of the Acoustical Society of America through the Frederick V. Hunt Postdoctoral Research Fellowship in Acoustics.

\section{Author contributions}

All authors contributed extensively to the analytical derivations, numerical calculations and the preparation of the manuscript. R.F. and A.K. contributed equally to this work.

\section{Additional information}

Competing financial interests: The authors declare no competing financial interests.

Reprints and permission information is available online at http://npg.nature.com/ reprintsandpermissions/

How to cite this article: Fleury, R. et al. Floquet topological insulators for sound. Nat. Commun. 7:11744 doi: 10.1038/ncomms11744 (2016)

(c) (i) This work is licensed under a Creative Commons Attribution 4.0 International License. The images or other third party material in this article are included in the article's Creative Commons license, unless indicated otherwise in the credit line; if the material is not included under the Creative Commons license, users will need to obtain permission from the license holder to reproduce the material. To view a copy of this license, visit http://creativecommons.org/licenses/by/4.0/ 